Article

\title{
Empirical and Numerical Analysis of Conduction and Induction Charging of Droplets in a Three-Electrode System
}

\author{
Adam Pelesz *(D) and Tomasz Czapka * \\ Department of Electrical Engineering Fundamentals, Faculty of Electrical Engineering, Wrocław University of \\ Science and Technology, 50-370 Wrocław, Poland \\ * Correspondence: adam.pelesz@pwr.edu.pl (A.P.); tomasz.czapka@pwr.edu.pl (T.C.)
}

Received: 20 November 2019; Accepted: 16 January 2020; Published: 18 January 2020

check for updates

\begin{abstract}
The charging process of water droplets in different electrification systems using empirical and numerical analysis is described. A simplified model based on capacitance distribution is proposed. The results of the experimental studies and numerical calculations enable comparison of the induction and conduction charging methods in terms of the droplet charging level, described by the $Q / m$ parameter. Research findings indicate that the conduction method in a 3-electrode system is the most effective among all the considered methods. It has been also shown that the application of a 2-electrode system under certain conditions provides greater $Q / m$ parameter values in comparison to the system with three electrodes using the induction method. The research results can be used to develop charging systems (e.g., nozzles) that produce streams with electrically charged liquid particles.
\end{abstract}

Keywords: electroaerosol; electrification of droplets; induction electrification

\section{Introduction}

Charging of aerosol particles is widely used in many technological processes [1-4]. Among the most common applications, one can mention agriculture (e.g., pesticide application and artificial pollination) [5-9], medicine (e.g., inhalation medications) [10,11], industry (e.g., painting, xerography, and food industries) [12-15], and others (e.g., thin film deposition, cosmetology, insecticide spraying) [16-18]. The use of electroaerosols improves the efficiency of a given process, for example providing more uniform object coverage and increasing the coating efficiency [19-21]. In general, there are several methods of aerosol charging, such as induction, conduction, corona discharge, postdispersion, and triboelectrification methods [2,12,13,22-27]. Induction and conduction charging methods are commonly used for conducting liquids. Furthermore, both methods can be implemented in two- or three-electrode systems. All mentioned methods, which require an external high voltage source, are schematically shown in Figure 1.

It should be noted that both conduction and induction methods are based on the principle of electrostatic induction. The key difference between them is the electric potential of the sprayed liquid. In the case of the induction method, the sprayed liquid is charged while being on ground potential. On the contrary, in the case of the conduction method, the liquid is charged at high potential (negative or positive). For a 2-electrode system, the induction and conduction methods are equivalent in terms of electric field distribution (i.e., if the influence of other surrounding objects, which may act as additional electrodes, are omitted). Moreover, in the case of a 3-electrode system, despite the same geometry (i.e., electrode configuration), there are some important differences, including in the electric field distribution, energy conversion, and the space charge effect. A more detailed description of the mentioned differences can be found in [25]. 
a)

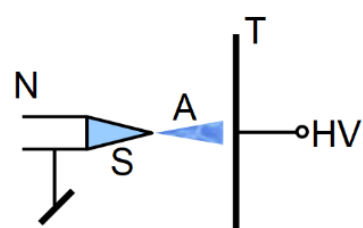

b)

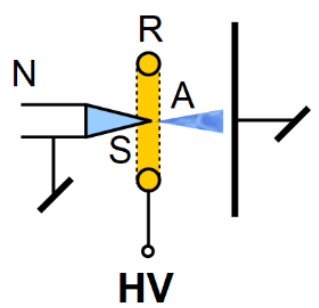

c)

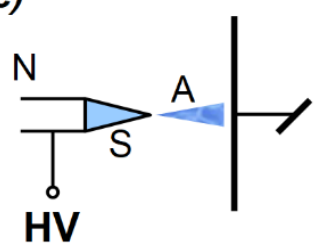

d)

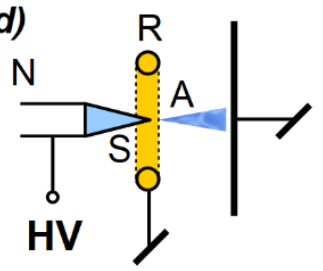

e)

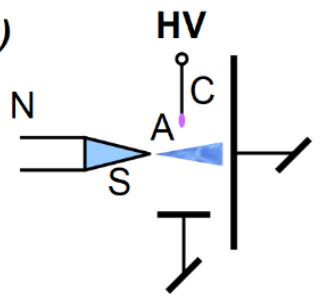

f)

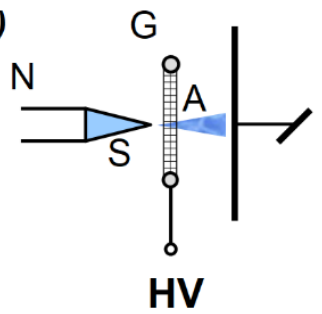

Figure 1. Schematic view of aerosol charging methods, requiring external high voltage source: (a) induction method in a 2-electrode system, (b) induction method in a 3-electrode system, (c) conduction method in a 2-electrode system, (d) conduction method in a 3-electrode system, (e) corona discharge method, and (f) postdispersion method. The main components of the system are: $\mathrm{N}$-conducting nozzle acting as electrode; S-stream of liquid; $\mathrm{A}$-aerosol; $\mathrm{T}$-target; $\mathrm{HV}$-high voltage source; $\mathrm{C}$-corona electrode; $\mathrm{R}$-ring electrode; $\mathrm{G}$ - grid electrode.

To compare different charging systems, a simple capacitance model and electrostatic numerical simulations have been proposed. Furthermore, the suggested concept seems to be sufficient to estimate the value of $Q / m$ at low liquid flow rates. The parameter $Q / m$ provides an unambiguous comparison of the individual charging systems from the point of view of their efficiency.

In our research, both arrangements are analyzed only in terms of the electrostatic field. In general, the induced surface charge density $q_{s i}$ of conducting objects is proportional to electric field $E$ acting on that object. For an object in a dielectric medium such as air, for which relative permittivity may be assumed as unitary $\varepsilon_{r}=1, q_{s i}$ can be given by:

$$
q_{s i}=\varepsilon_{0} E .
$$

To obtain the total charge $Q_{i}$ induced on the object (e.g., a droplet), one should integrate the charge over the entire surface $s$ :

$$
Q_{i}=\int_{s} q_{s i} \mathrm{~d} s .
$$

For a conducting object, the induced charge $Q_{i}$ is a result of the object-environment capacitance $C$ and the resulting potential difference $V$ :

$$
Q_{i}=V \cdot C .
$$

\section{Capacitance Distribution in the Charging Systems}

When spraying with water and aqueous solutions (e.g., pesticides), a droplet can be effectively treated as a conducting object. This is due to the fact that the droplet is affected by an electric field longer than its material charge-transfer time constant $\tau$ [1,2]. For the mentioned liquids, this time constant can be lower than $1 \mu \mathrm{s}$ [2]. As can be seen from Equation (3), the electrical capacitance of the system used during the charging process is the main factor (i.e., apart from the electric field related to the potential difference $V$ ) that directly influences the value of the charge induced on the droplet. The capacitance distributions in two- and three-electrode systems are schematically shown in Figure 2. 
a)

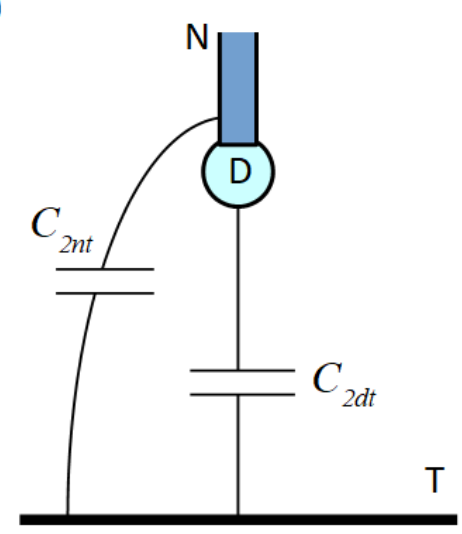

b)

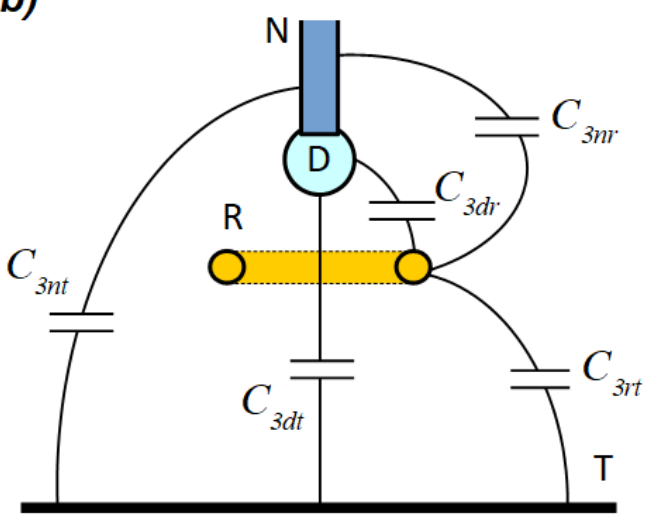

Figure 2. Capacitance distribution in a (a) 2-electrode system and (b) 3-electrode system. Note: T-target; $\mathrm{R}$-ring electrode; $\mathrm{D}$-droplet; $\mathrm{N}$-nozzle; $\mathrm{C}_{2 n t}$-capacitance between nozzle and target in a 2-electrode system; $C_{2 d t}$-capacitance between droplet and target in 2-electrode system; $C_{3 n t}$-capacitance between nozzle and target in a 3-electrode system; $C_{3 n r}$-capacitance between nozzle and ring electrode in a 3-electrode system; $C_{3 d r}$-capacitance between droplet and ring electrode in a 3-electrode system; $C_{3 d t}$-capacitance between droplet and target in a 3-electrode system; $C_{3 r t}$-capacitance between ring electrode and target in a 3-electrode system.

In the presented model, the influence of any other objects potentially appearing in the surrounds of the system was omitted. To simplify the analysis, it was assumed that all other objects are far from the electrification setup. Thus, capacitances associated with such objects were negligibly small and excluded from further analysis. In the real setup, however, the impact of such capacitances may be significant (especially in a 2-electrode system when the target is far away from a droplet, because the capacitance $C_{2 d t}$ can then be comparable to the droplet-environment capacitance). It should be noted that charge induced on the droplet depends only on capacitances associated with the droplet (i.e., $C_{2 d t}, C_{3 d t}, C_{3 d r}$ ). The capacitances $C_{2 n t}, C_{3 n t}, C_{3 n r}$, and $C_{3 r t}$ do not directly influence the droplet charging process. However, those additional capacitances were marked because they constitute part of the analyzed systems. It was also assumed that the conductive droplet was always in contact with the conductive nozzle (i.e., a droplet and a nozzle together form a single electrode), and thus droplet potential was well determined.

In a 2-electrode system, regardless of the charging method, droplet-induced charge $Q_{2 e l}$ can be given by:

$$
Q_{2 e l}=V_{2 d t} \cdot C_{2 d t}=V \cdot C_{2 e l}
$$

where $V_{2 d t}$ is the potential difference between a droplet and a target in a 2-electrode system (i.e., the charging voltage $V), Q_{2 e l}$ is the induced charge in a case of a 2-electrode system, and $C_{2 e l}$ is the total capacitance associated with a droplet in the 2-electrode system (in this case $C_{2 e l}=C_{2 d t}$ from Figure 2).

In general, for a 3-electrode system, the expression for induced charge $Q_{3 e l}$ is given by:

$$
Q_{3 e l}=V_{d r} \cdot C_{3 d r}+V_{3 d t} \cdot C_{3 d t}
$$

where $V_{d r}$ is the potential difference between a droplet and a ring electrode, and $V_{3 d t}$ is the potential difference between a droplet and a target in a 3-electrode system.

In the case of the induction method, there is no potential difference between a droplet and a target $V_{3 d t}=0$, and the potential difference between a droplet and a ring electrode is equal to the charging voltage $V_{d r}=V$. Therefore, the induced charge is:

$$
Q_{3 e l \_i n d}=V_{d r} \cdot C_{3 d r}=V \cdot C_{3 e l} \text { ind } .
$$


On the other hand, in the case of conduction method, the potential difference between a droplet and a target is equal to the potential difference between a droplet and a ring electrode, and equal to the charging voltage (i.e., $V_{3 d t}=V_{d r}=V$ ). Consequently, the induced charge is given by:

$$
Q_{3 e l \_c o n d}=V \cdot C_{3 d r}+V \cdot C_{3 d t}=V \cdot\left(C_{3 d r}+C_{3 d t}\right)=V \cdot C_{3 e l \_c o n d},
$$

where $Q_{3 e l \_ \text {ind }}$ is the induced charge in a case of a 3-electrode system and induction method, $Q_{3 e l \_c o n d}$ is the induced charge in a case of a 3-electrode system and conduction method, $C_{3 e l}$ ind is the total capacitance associated with a droplet in the 3-electrode system for induction method (i.e., $C_{3 e l}$ ind $=C_{3 d r}$ from Figures 2 and 3), and $C_{3 e l}$ cond is the total capacitance associated with a droplet in the 3-electrode system for induction method (i.e., $C_{3 e l \_c o n d}=\left(C_{3 d r}+C_{3 d t}\right)$ from Figures 2 and 3).
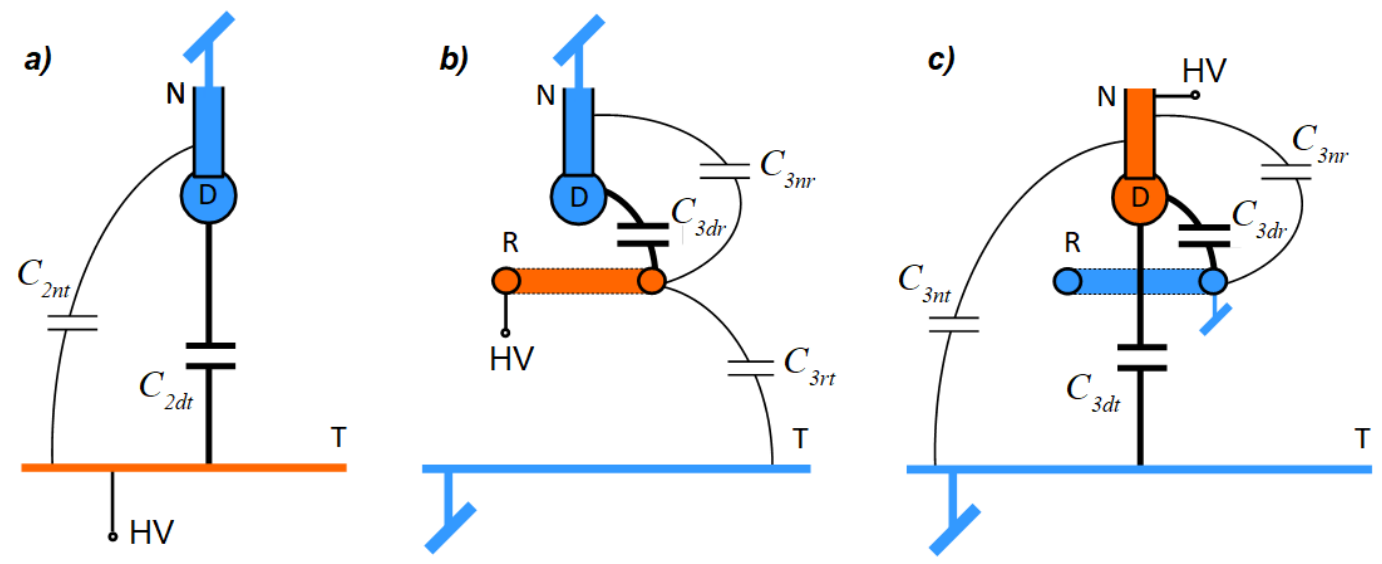

Figure 3. Effective capacitance distribution: (a) induction method in a 2-electrode system, (b) induction method in a 3-electrode system, and (c) conduction method in a 3-electrode system. Capacitances associated directly with droplets are marked with a thicker line.

Considering that a capacitance is a function of the geometry of the electrodes and the dielectric properties of the medium between them, it is important to note that the potential difference between electrodes does not affect the capacitance distribution in the system or the value of these capacitances. However, if there is no potential difference between the electrodes, no charge can be induced, so the effect of this capacitance can be omitted. The concept of so-called effective capacitance distribution only applies to the capacitances between electrodes with a potential difference. Alternatively, it can be assumed that the electrodes on the same potential are in a galvanic contact with each other, forming one single electrode. The concept of capacitance between such electrodes becomes virtually meaningless.

Effective capacitance distributions for induction and conduction methods are presented in Figure 3. In the following considerations, it is assumed that 3-electrode systems have the same geometry.

The capacitance distribution in a 2-electrode system is the same regardless of the charging method. In the case of a 3-electrode system, capacitance values do not change, however the effective capacitance distribution varies because of the change in the potential values. In a 3-electrode system where the induction method is due to the lack of potential difference between a droplet and a target, the capacitance $C_{d t}$ can be omitted, as shown in Figure 3b.

As capacitances have always positive values, it is obvious that:

$$
\left(C_{3 d r}+C_{3 d t}\right)>C_{3 d r}
$$

Therefore, in a 3-electrode system, based on Equations (6) and (7):

$$
C_{3 e l \_c o n d}>C_{3 e l \_i n d} \text {. }
$$


Thus, if the same value of voltage is applied, it can be shown from Equation (3) that:

$$
Q_{3 e l \_c o n d}>Q_{3 e l \_i n d} \text {. }
$$

If $C_{3 d t}<<C_{3 d r}$ (e.g., the target is far away from the nozzle, and the ring electrode is close to the nozzle), charges induced in the particular systems can be given by:

$$
Q_{3 e l \_c o n d} \cong Q_{3 e l_{\text {ind }}} \text {. }
$$

It should be noted that the capacitances $C_{2 d t}$ and $C_{3 d t}$ are not equal. Geometries in a 2-electrode system and a 3-electrode one are different due to the presence of the ring electrode. The capacitance $C_{2 d t}$ is related to the total droplet surface. However, in the case of a 3-electrode system, the capacitance $C_{3 d t}$ is associated only with part of the droplet surface because of the presence of the $C_{3 d r}$ capacitance, as shown in Figure 2b. Because the capacitance is directly proportional to the electrode surface area, the relation between these two capacitances is:

$$
C_{2 d t}>C_{3 d t}
$$

In the case of the conduction method, the electric field associated with a droplet affects the target or the ring electrode. If the ring is closer to the droplet than the target it can be hypothesized that:

$$
\left(C_{3 d r}+C_{3 d t}\right)>C_{2 d t}
$$

Considering the conduction method in Equation (13):

$$
C_{3 e l \_c o n d} \geq C_{2 e l},
$$

Thus, assuming that the same voltage is applied:

$$
Q_{3 e l \_c o n d} \geq Q_{2 e l} \text {. }
$$

The case $Q_{3 e l \_c o n d} \cong Q_{2 e l}$ refers to the situation where the ring electrode is far away from the nozzle in the 3-electrode system with the conduction method, and thus the electric field distribution in that system is similar to that in the 2-electrode system.

In general, the value of $C_{3 d r}$ can be greater, smaller, or equal to the value of $C_{2 d t}$, so there are possible electrode configurations where:

$$
C_{2 d t}>C_{3 d r}
$$

In the case of the induction method, Equation (16) can be given by:

$$
C_{2 e l}>C_{3 e l \_i n d}
$$

Thus, assuming that the same voltage is applied:

$$
Q_{2 e l}>Q_{3 e l \_i n d} \text {. }
$$

Equations (16)-(18) are only valid for one of three possible situations (e.g., $C_{2 d t}>C_{3 d r}$ ), which occur when the surface of the ring electrode is much smaller than the target surface. As a consequence of the above-mentioned remark, there may be some electrode configurations in which charge induced in a 2-electrode system is higher than charge induced with the induction method in a 3-electrode system for the same applied voltage. 


\section{Computer Simulations of the Droplet Charging Process}

To show the electric field distribution for cases described in the previous section, numerical finite element method (FEM) simulations were performed in the COMSOL Multiphysics 4.3 software using 2D axisymmetric geometry. Thus, the presented model employs an analysis of the distribution of electrical capacitances in the electrification system with different methods of droplets charging. The area of the calculated electric field distribution was restricted to a cylinder with a height of $150 \mathrm{~mm}$ and a diameter of $100 \mathrm{~mm}$. On the external boundaries of the model (excluding the grounded floor, i.e., the target) only the tangential component of the electric field could have existed. The permittivity of the medium filling the space was assumed to be $\varepsilon_{r}=1$ (i.e., air). Electric field distributions for induction and conduction methods in the 3-electrode system are presented in Figures 4 and 5.

a)

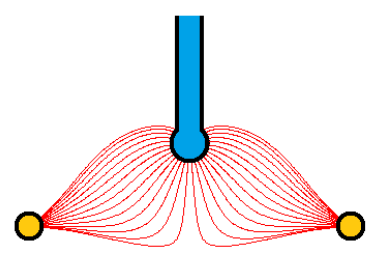

b)

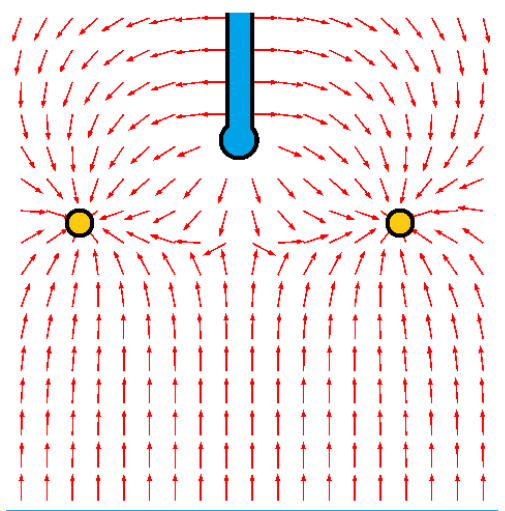

Figure 4. Sketch of electric field lines associated with the droplet (a) and direction of the electric field (b) for the induction method. A target and a nozzle with a droplet are grounded and the polarity of the voltage applied to the ring electrode is negative, so the induced charge is positive.

a)

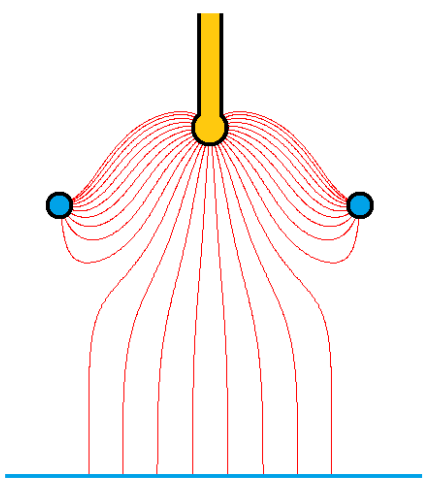

b)

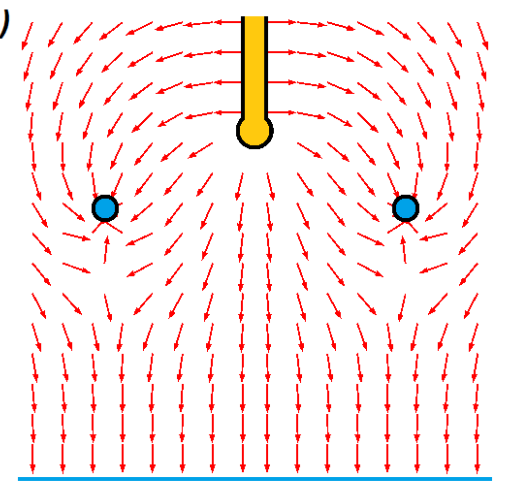

Figure 5. Sketch of electric field lines associated with the droplet (a) and direction of the electric field (b) for the conduction method. A ring electrode and a target are grounded and the polarity of the voltage applied to the droplet is positive, so the induced charge is also positive.

In Figure 5, it can be seen that in the case of the conduction method, the electric field lines (associated with the droplet) are linked to the ring electrode and the target. In contrast, in the case of the induction method, the electric field lines (associated with the droplet) are linked only to the ring electrode, as shown in Figure 4.

To illustrate the influence of different electrode configurations on the charging process, another FEM model was created using 2D axisymmetric geometry. The geometry of the assumed setup is shown in Figure 6. The simulation area was again restricted to a cylinder with a height of $500 \mathrm{~mm}$ and a diameter of $200 \mathrm{~mm}$. Other parameters of the simulation (i.e., external boundary condition, medium permittivity) were unchanged in comparison to the simulation described previously (Figures 4 and 5). 


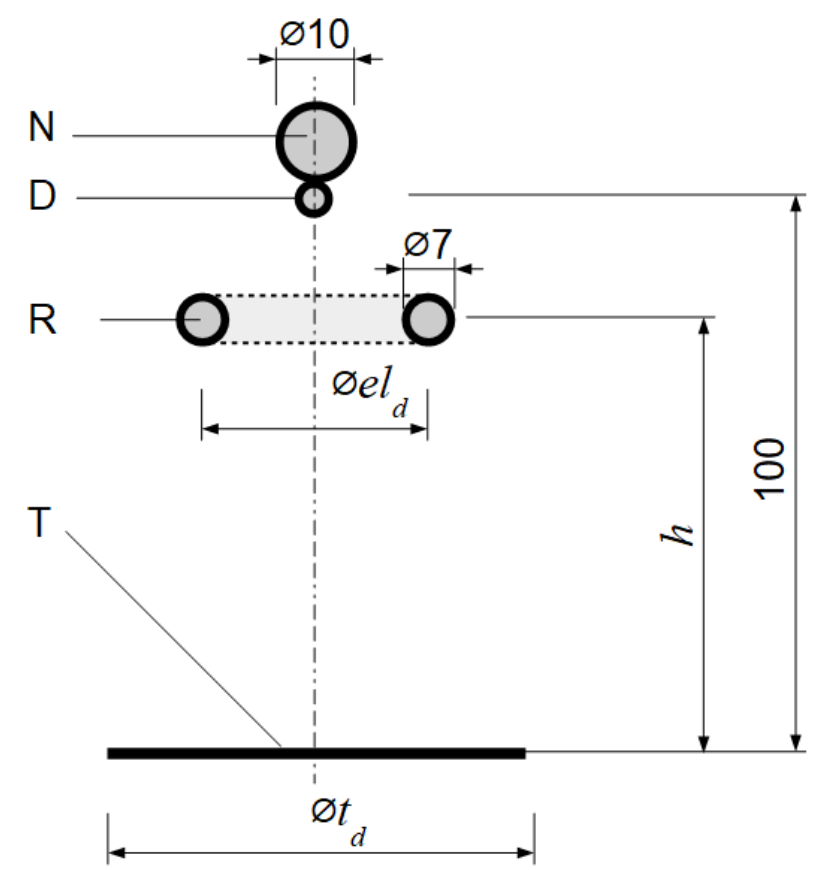

Figure 6. Sketch of the droplet charging geometry used in finite element method (FEM) numerical simulation. Note: $\mathrm{N}$-a nozzle (e.g., conducting needle with water supply); D—droplet with a diameter of $2 \mathrm{~mm}$. All dimensions are in $\mathrm{mm}$.

In the analyzed model, the ring electrode can be in a position below $(h<100 \mathrm{~mm})$ or above $(h>100 \mathrm{~mm})$ the droplet, because the position of the ring electrode does not affect the capacitance distribution in the system (presented in Figures 1 and 2), rather only slightly changes the values of these capacitances.

In the described above model, various scenarios were represented by changing the diameter of the ring electrode $e l_{d}$ or the target $t_{d}$. The following cases were analyzed:

A. $\quad e l_{d}=40 \mathrm{~mm}, t_{d}=20 \mathrm{~mm}$ (i.e., a narrow ring electrode and a small target);

B. $e l_{d}=40 \mathrm{~mm}, t_{d}=200 \mathrm{~mm}$ (i.e., a narrow ring electrode and a big target);

C. $e l_{d}=80 \mathrm{~mm}, t_{d}=200 \mathrm{~mm}$ (i.e., a wide ring electrode and a big target).

The terms "small", "big", "narrow", and "wide" should be treated in a relative rather than an absolute manner.

In analyzed situations, the droplet-target distance was kept constant and equal to $100 \mathrm{~mm}$. In the physical system realization, this parameter also plays an important role. However, when the droplet is far away from the target and the ring electrode is close to the nozzle, the capacitance $C_{3 d t}$ should be negligible, and then both methods (i.e., conduction and induction) should give similar results. The presented analysis focuses only on cases where the nozzle is relatively close to the grounded target. In all three cases, the ring electrode height $h$ was changed in the range of $25-200 \mathrm{~mm}$. The total capacitance between the droplet and the environment was numerically calculated, and the obtained values were divided by the droplet-environment capacitance for the 2-electrode system, which is the same regardless of the charging method.

Taking into account the capacitance distributions shown in Figure 3, particular capacitances can be given by:

$$
\begin{gathered}
C_{3 e l \_c o n d}=\left(C_{3 d r}+C_{3 d t}\right), \\
C_{3 e l \_i n d}=C_{3 d r}, \\
C_{3 d t}=\left(C_{3 e l \_c o n d}-C_{3 e l \_i n d}\right) .
\end{gathered}
$$


It should be also pointed out that induced charge is directly proportional to corresponding capacitances, namely in Equations (3), (4), (6), and (7). Capacitances $C_{2 e l}, C_{3 e l \_c o n d}$, and $C_{3 e l \_ \text {ind }}$ are called droplet capacitances because of their corresponding charging methods. Values of capacitances $C_{3 e l}$ cond and $C_{3 e l}$ ind relative to $C_{2 e l}$ for different electrification conditions are shown in Figure 7 (i.e., case A: a narrow ring electrode and a small target), Figure 8 (i.e., case B: a narrow ring electrode and a big target), and Figure 9 (i.e., case C: a wide ring electrode and a big target).

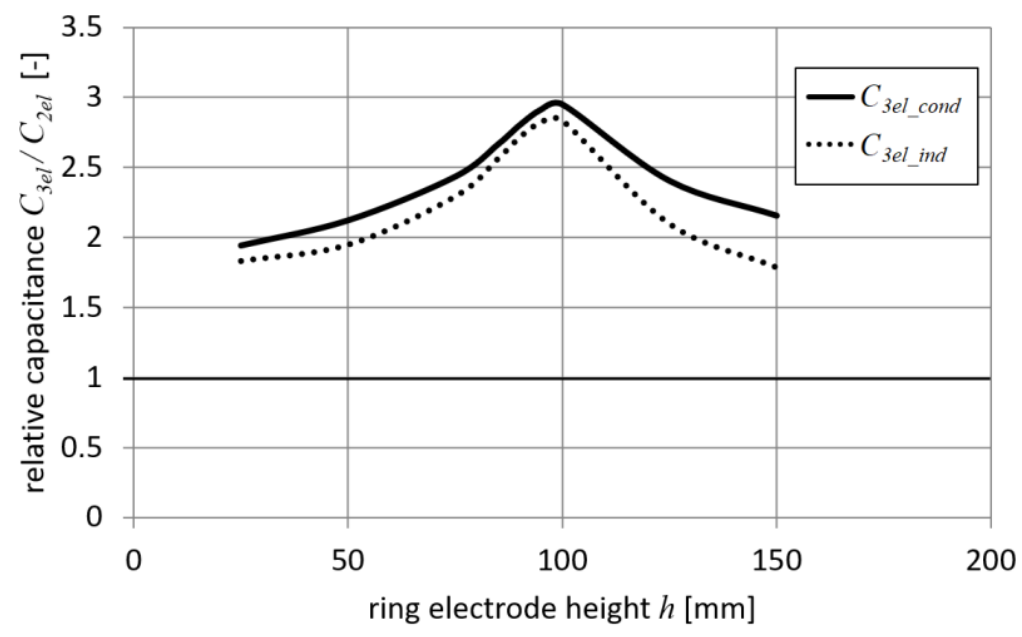

Figure 7. Droplet capacitance $C_{3 e l}$ values for induction and conductive charging methods, relative to the capacitance of the 2-electrode system $C_{2 e l}$, as a function of ring electrode height $h$. Simulation parameters: $r_{d}=40 \mathrm{~mm}, t_{d}=20 \mathrm{~mm}$ (i.e., narrow ring electrode and a small target).

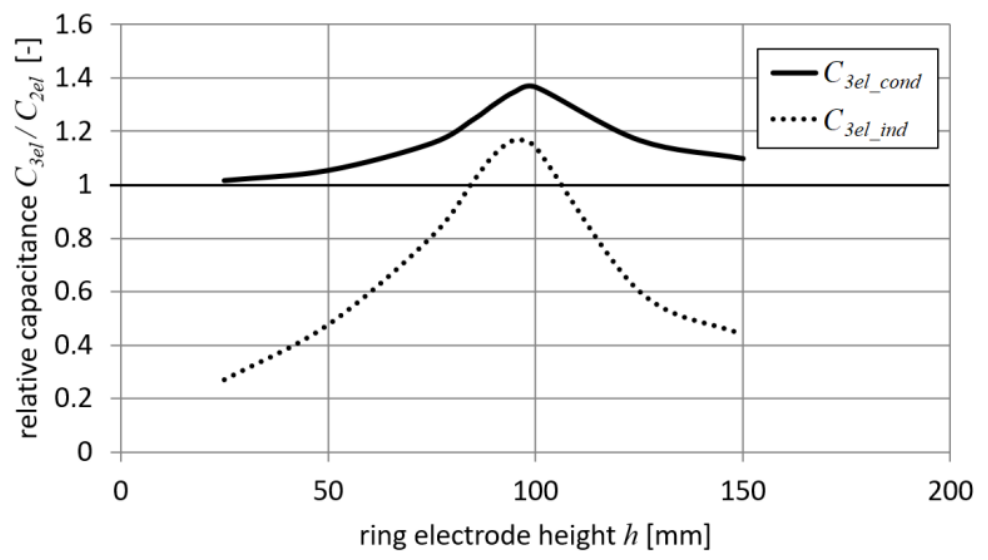

Figure 8. Droplet capacitance $C_{3 e l}$ values for induction and conductive charging methods, relative to the capacitance of the 2-electrode system $C_{2 e l}$, as a function of ring electrode height $h$. Simulation parameters: $r_{d}=40 \mathrm{~mm}, t_{d}=200 \mathrm{~mm}$ (i.e., narrow ring electrode and a big target).

Results for a setup with a narrow ring electrode $\left(r_{d}=40 \mathrm{~mm}\right)$ and a small target $\left(t_{d}=20 \mathrm{~mm}\right)$ are shown in Figure 7. It can be seen from Figure 7 that both droplet capacitances $C_{3 e l \_c o n d}$ and $C_{3 e l \_}$ind have similar values. The differences are most significant when the ring electrode is high above the droplet ( $h>120 \mathrm{~mm}$ ). In this case, $C_{3 d t}$ is small and $C_{3 d r}$ is dominant when conduction charging is considered, such as in Equations (20) and (21). The maximum values of capacitances $C_{3 e l \_c o n d}$ and $C_{3 e l \_ \text {ind }}$ were obtained for the height of the ring electrode $h$ equal to $100 \mathrm{~mm}$ (i.e., when the ring electrode is near the droplet). These capacitance values for 3-electrode systems were about 3 times higher than the capacitance $C_{2 e l}$ calculated for the setup with two electrodes. Based on the presented results, similar values of the induced charge for both methods using a 3-electrode system can be expected. The presented case corresponds to a system with a well-designed ring electrode. 


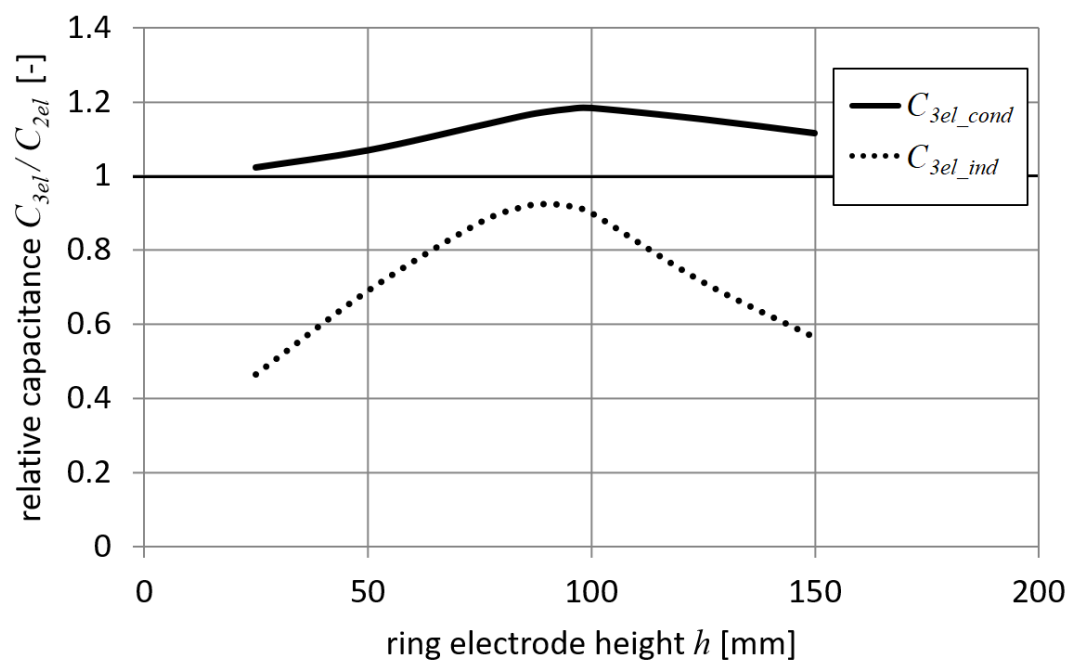

Figure 9. Droplet capacitance $C_{3 e l}$ values for induction and conductive charging methods, relative to the capacitance of the 2-electrode system $C_{2 e l}$, as a function of ring electrode height $h$. Simulation parameters: $r_{d}=80 \mathrm{~mm}, t_{d}=200 \mathrm{~mm}$ (i.e., a wide ring electrode and a big target).

Results for the setup with a narrow ring electrode $\left(r_{d}=40 \mathrm{~mm}\right)$ and a big target $\left(t_{d}=200 \mathrm{~mm}\right)$ are shown in Figure 8 . Figure 8 shows that the capacitance $C_{3 e l \_c o n d}$ always significantly exceeds $C_{3 e l \_i n d}$, especially when the ring electrode is far away from the droplet position. This effect indicates that for conduction charging, the influence of both capacitances (i.e., $C_{3 d r}$ and $C_{3 d t}$ ) is comparable. Based on these results and Equation (21), it can be concluded that an increase in the distance between the ring electrode and the droplet leads to an increase in the capacitance $C_{3 d t}$ influence. It was also found that there are some positions of the ring electrode $h$ for which the capacitance $C_{3 e l \_i n d}$ is lower than the droplet capacitance of a 2-electrode system $C_{2 e l}$ (i.e., values lower than 1 in Figure 8). Moreover, it was observed that for a certain narrow range of height $h$ (i.e., $85<h<105 \mathrm{~mm}$ ), a 3-electrode system with induction charging provides higher $Q / m$ values (i.e., values greater than 1 in Figure 8).

Results for the setup with a wide ring electrode $\left(r_{d}=80 \mathrm{~mm}\right)$ and a big target $\left(t_{d}=200 \mathrm{~mm}\right)$ are shown in Figure 9. As can be seen in Figure 9, the capacitance $C_{3 e l}$ cond is always significantly greater than $C_{3 e l \_i n d}$, which is a similar situation to the previous case. In addition, it can be noticed that the induction charging method in a 3-electrode configuration is always less efficient than electrification in the 2-electrode system (i.e., values lower than 1 in Figure 9). The discussed case corresponds to a system with a poorly designed electrode arrangement.

The following findings can be made for all analyzed cases:

- droplet capacitance in a 3-electrode system is the highest when the ring electrode is slightly under the droplet;

- droplet capacitance in the 3-electrode conduction method $C_{3 e l}$ cond is always greater than in the 3-electrode induction method $C_{3 e l}$ ind or 2-electrode system $C_{2 e l}$;

- for certain electrode configurations (i.e., the position of the ring electrode), droplet capacitance in the 3-electrode induction method $C_{3 e l}$ ind is lower than in the 2-electrode system $C_{2 e l}$, even if the ring electrode is at the same height as the droplet (Figure 9);

- droplet capacitance in the 3-electrode conduction method $C_{3 e l}$ cond is less dependent on the ring electrode position.

Considering that the induced charge is directly proportional to droplet capacitance, as stated in Equation (3), and assuming the same value of applied voltage for 2- and 3-electrode systems, the above-mentioned observations lead to the conclusion that a 3-electrode conduction charging method always gives higher values of droplet charge than a 3-electrode induction method or a 2-electrode 
system. Moreover, the 3-electrode induction method may sometimes be less efficient in terms of the induced charge than the 2-electrode system.

It should also be noted that the above examples were given to illustrate the influence of different electrode arrangements on the charging levels of droplets, and are not related to any real geometry. When designing the electrode system, other factors should also be taken into account (e.g., electrical strength of the system).

In the real dynamic case of the charging process, the droplet (i.e., water or other liquid) is deformed due to the electric field. Since in each setup the electric field is different (even at the same voltage), the droplet deformation may be different. This effect causes further changes in the electric field distribution, and thus leads to a different induced charge. Other factors affecting the value of the $Q / m$ ratio are space charge [28] and flow rate, as well as sprayed liquid properties [29]. The charged droplets may also shield the sprayed liquid from the electric field, reducing the charging level [30]. It should also be mentioned that the charge of a droplet is limited due to the electric field strength of the medium or due to the Rayleigh limit [1,2]. For that reason, one droplet can separate into smaller droplets [1,2], and very complex numerical simulation techniques (i.e., including particle tracking, moving mesh, and computational fluid dynamics CFD) [31-34] are often used to model the droplet charging process.

\section{Measurements and Calculations of the $Q / m$ Parameter}

To confirm the validity of the previous theoretical considerations, a simple experimental setup for the measurements of the $Q / m$ parameter was proposed. The main parts of the setup were the pump (P) equipped with a rubber pipe (RP) for pumping water, a metallic needle as a nozzle (N) with a needle holder $(\mathrm{NH})$ and a conducting ring electrode (R) (for which height above the ground $h$ was adjusted during the experiment), and a Faraday cup (F) in a grounded shield (GS). The Faraday cup was almost completely shielded and only had a hole allowing the charged droplets to enter it. In the charging process, a high-voltage DC power supply was used. The switch $S$ was used to change the electrification method (i.e., induction or conduction). The measurement setup is shown in Figure 10. The capacitance of the cable and Faraday cup $C_{n}$ was equal to $1.18 \mathrm{nF}$. The voltage on $C_{n}$ was measured using electrometer.

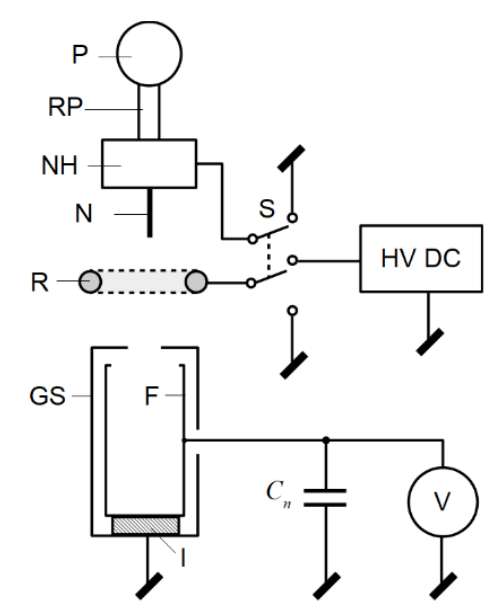

Figure 10. Scheme of the measurement setup. Note: $\mathrm{P}$-pump; RP-rubber pipe; N-nozzle; NH-needle holder; S-switch; R-ring electrode; F-Faraday cup; GS-grounded shield; HV DC—high-voltage DC power supply.

The diameter and the mass of the droplets formed during the charging process were equal to $2.7 \mathrm{~mm}$ and $10.3 \mathrm{mg}$, respectively. The ratio of droplet generation was equal to 100 droplets per minute to avoid a shielding effect from other charged droplets. The applied voltage was equal to about $5 \mathrm{kV}$ (positive for the conduction method and negative for the induction method), so the droplet charge was always positive. A specific voltage level was chosen to avoid deformation of the liquid droplet or 
droplet breakdown into smaller droplets. For this reason, the obtained values of $Q / m$ were relatively low. Distilled water with a conductivity of $1.4 \mu \mathrm{S} / \mathrm{cm}$ (measured at $23^{\circ} \mathrm{C}$ ) was used in the studies.

Based on the measurement setup, another FEM simulation model was proposed, as outlined in Figure 11. The simulation area was a cylinder measuring $100 \mathrm{~mm}$ in height and $1000 \mathrm{~mm}$ in diameter. Other simulation parameters (i.e., external boundary condition, medium permittivity) were unchanged in relation to the previously described simulations. The total induced charge on droplet $Q_{d}$ was numerically calculated. To obtain the $Q / m$ parameter, the value of $Q_{d}$ was divided by the experimentally measured droplet mass.

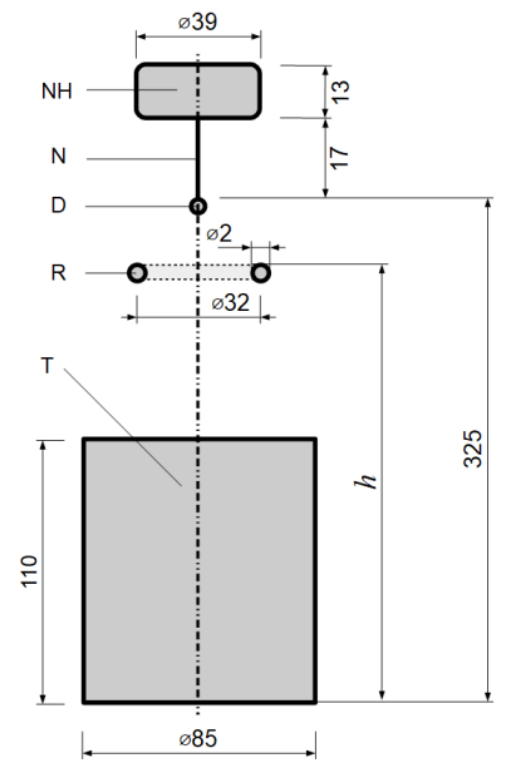

Figure 11. View of the model used in finite element method (FEM) computer simulation, based on the measurement setup shown in Figure 10. Note: N-nozzle (needle); NH—needle holder; D—water droplet; $\mathrm{R}$-ring electrode; $\mathrm{T}$-target. The droplet diameter was equal to $2.7 \mathrm{~mm}$. All dimensions are in $\mathrm{mm}$.

The first experiment was carried out for a 2-electrode setup (i.e., without the ring electrode). The measured and calculated values of the $Q / m$ parameter were equal to $15.6 \mu \mathrm{C} / \mathrm{kg}$ and $20.4 \mu \mathrm{C} / \mathrm{kg}$, respectively. This means that the calculated $(\mathrm{Q} / \mathrm{m})_{2 e l \_s i m}$ was 1.31 times greater than the measured $(Q / m)_{2 e l \_e x p}$.

In the next part of the experimental study, the ring electrode was introduced into the system, which enabled the charging of droplets in a 3-electrode configuration. The height of the ring electrode $h$ was in the range of 180 to $330 \mathrm{~mm}$. The results of $Q / \mathrm{m}$ measurements and simulation-based calculations are shown in Figure 12. It can be seen from Figure 12 that the differences between numerically calculated and measured values do not exceed $20 \%$. 


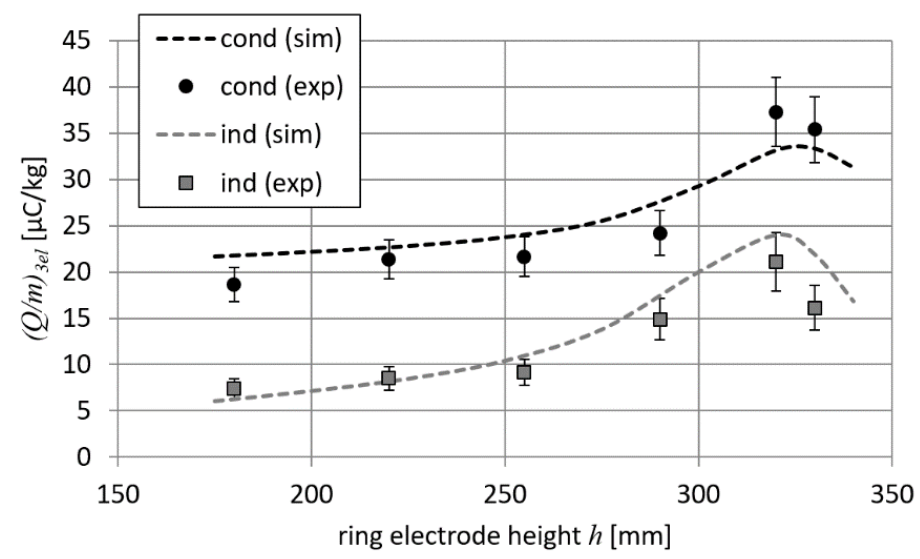

Figure 12. Measured ("exp") and calculated ("sim") $(Q / m)_{3 e l}$ parameter values for both electrification methods in the 3-electrode system, as a function of the ring electrode height $h$.

Figure 13 shows the measured and calculated $(Q / m)_{3 e l}$ parameter values for conduction and induction methods in the 3-electrode system divided by the $(Q / m)_{2 e l}$ parameter values obtained in 2-electrode systems (i.e., measured and calculated for corresponding cases).

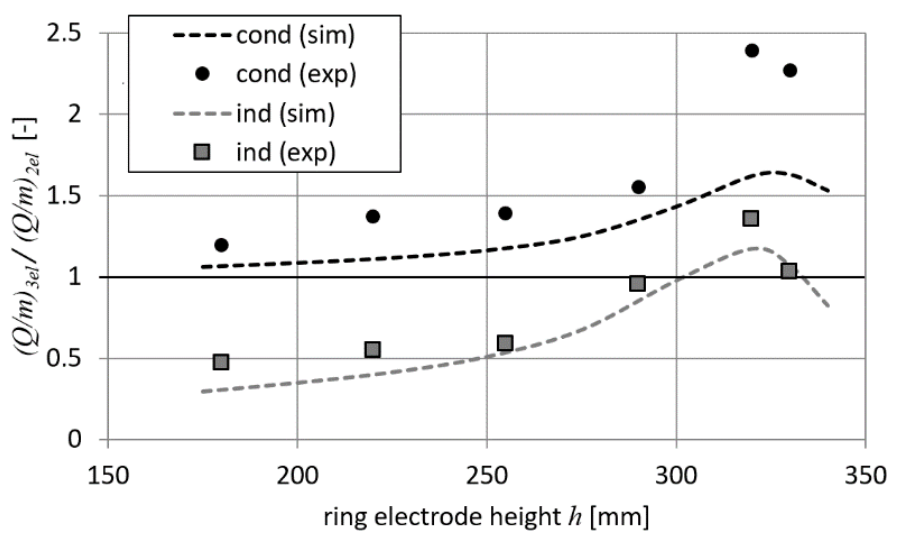

Figure 13. Measured ("exp") and calculated ("sim") $(Q / m)_{3 e l}$ parameter values for both electrification methods ("ind" and "cond") in a 3-electrode system, relative to $Q / m$ of a 2-electrode system, as a function of a the ring electrode height $h$. Simulation results relative to $(Q / m)_{2 e l \_s i m}$, empirical results relative to $(Q / m)_{2 e l \_e x p}$.

It can be seen from Figure 13 that the differences between simulation and experimental results are clearly visible. This is the result of different values of $(Q / m)_{2 e l}$ being obtained from measurements and calculations $(15.6 \mu \mathrm{C} / \mathrm{kg}$ and $20.4 \mu \mathrm{C} / \mathrm{kg}$, respectively). It is also clear that for most of the tested ring electrode positions, the induction method in a 3-electrode system is less efficient than a 2-electrode charging system (i.e., relative values of $Q / m$ are lower than 1, as shown in Figure 13). Only for the case in which a ring electrode is close to the droplet is the induction method better than a 2-electrode system in terms of charging efficiency. On the other hand, the conduction method in a 3-electrode system is always more effective than a 2-electrode charging system (i.e., relative values of $Q / m$ parameter are greater than 1, as presented in Figure 13). The obtained results are consistent with those shown in Figure 8. It should be noted that according to the simulation data, in a 3-electrode system the $Q / m$ parameter should be less dependent on the ring electrode position in the case of conduction method than in the system with the induction method. However, the results of the measurements show that $Q / m$ greatly increases in the case of the conduction method when the ring electrode is close to the droplet.

Ratios of $Q / m$ parameters for both charging methods are presented in Figure 14. It can be noticed in Figure 14 that the conduction method is always more effective than the induction method 
(i.e., 1.5-2.5 times greater $Q / m$ parameter values are obtained). The results of the numerical simulation and measurements are consistent with each other. A noticeable difference for cases when the ring electrode is far from the droplet may be due to the external factors (i.e., the appearance of additional coupling capacitances).

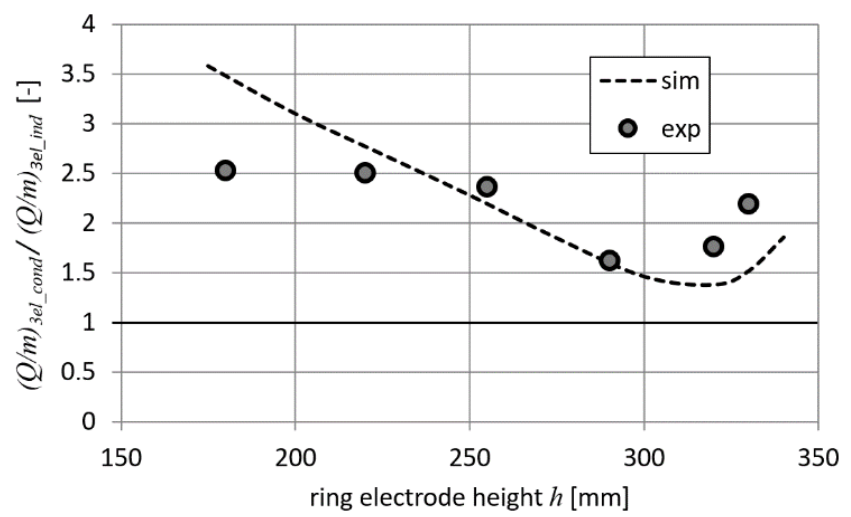

Figure 14. Ratio of measured ("exp") and calculated ("sim") $Q / m$ parameter values for both conduction $(Q / m)_{3 e l \_c o n d}$ and induction $(Q / m)_{3 e l \_i n d}$ methods in the 3-electrode system.

\section{Discussion and Conclusions}

The proposed simplified model based on the capacitance distribution in different electrification systems can be used to study the process of electrification of liquid droplets. For a 2-electrode system, only the capacitance between the nozzle and the target is responsible for the charging level of the droplets. In the 3-electrode systems, the capacitance distribution is more complex. In the case of the induction method, the value of the charge induced on the droplet depends only on the capacitance between the droplet and the ring electrode. When the conduction method is considered, the capacitance between the droplet and the target has an additional influence on the charging level. The total capacitance responsible for inducing charge on the droplet is the highest in the latter case, making the 3-electrode system with the conduction method the most efficient from the point of view of charging level, assuming that the same voltage is applied in all systems.

Based on the model, it is possible to determine the $Q / m$ parameter describing the efficiency of the electrification systems. It can be noticed that in a 3-electrode system, the conduction charging method can give significantly higher $Q / m$ values than in the induction method, especially when the grounded target is close to the nozzle, which greatly affects the electric field near the droplet. Similar findings were established during experiments. A significant coherence of simulation and measurement results confirms the validity of the proposed model, which can be helpful when designing a real-life charging system (i.e., setting the distance between the ring electrode and the nozzle).

It has also been proven that for a specific electrode configuration using the induction method, the 2-electrode system is more effective than the 3-electrode setup, and in this case, the measured values of the $Q / m$ parameter were even smaller than for the 3-electrode system.

Future studies will focus on developing a model that considers the dynamic processes occurring during droplet electrification, including the screening effect and deformation of droplets.

Author Contributions: Conceptualization, A.P.; methodology, A.P. and T.C.; validation, A.P. and T.C.; formal analysis, A.P. and T.C.; investigation, A.P. and T.C.; resources, T.C.; writing-original draft preparation, A.P.; writing-review and editing, A.P. and T.C.; visualization, A.P.; funding acquisition, T.C. All authors have read and agreed to the published version of the manuscript.

Funding: This research received no external funding. This work was financed by a statutory activity subsidy from the Polish Ministry of Science and Higher Education (PMSHE) for the Department of Electrical Engineering Fundamentals of Wroclaw University of Science and Technology.

Conflicts of Interest: The authors declare no competing financial interest. 


\section{References}

1. Chang, J.S.; Kelly, A.J.; Crowley, J.M. Handbook of Electrostatic Processes; CRC Press: Boca Raton, FL, USA, 1995.

2. Bailey, A.G. Electrostatic Spraying of Liquids; Taunton Research Studies Press: London, UK, 1988.

3. Jaworek, A.; Sobczyk, A.T.; Krupa, A. Electrospray application to powder production and surface coating. J. Aerosol Sci. 2018, 125, 57-92. [CrossRef]

4. Cloupeau, M.; Prunet-Foch, B. Electrostatic spraying of liquids: Main functioning modes. J. Electrost. 1990, 25, 165-184. [CrossRef]

5. Castle, G.P.; Inculet, I.I. Space charge effects in orchard spraying. IEEE Trans. Ind. Appl. 1983, IA-19, 476-480. [CrossRef]

6. Patel, M.K. Technological improvements in electrostatic spraying and its impact to agriculture during the last decade and future research perspectives-A review. Eng. Agric. Environ. Food. 2016, 9, 92-100. [CrossRef]

7. Matthews, G.A. Electrostatic spraying of pesticides: A review. Crop Prot. 1989, 8, 3-15. [CrossRef]

8. Law, S.E. Agricultural electrostatic spray application: A review of significant research and development during the 20th century. J. Electrost. 2001, 51-52, 25-42.

9. Bechar, A.; Shmulevich, I.; Eisikowitch, D.; Vaknin, Y.; Ronen, B.; Gan-Mor, S. Modeling and experiment analysis of electrostatic date pollination. Trans. ASAE 1999, 42, 1511-1516. [CrossRef]

10. Balachandran, W.; Machowski, W.; Gaura, E.; Hudson, C. Control of drug aerosol in human airways using electrostatic forces. J. Electrost. 1997, 40, 579-584. [CrossRef]

11. Wong, J.; Chan, H.K.; Kwok, P.C.L. Electrostatics in pharmaceutical aerosols for inhalation. Ther. Deliv. 2013, 4, 981-1002. [CrossRef]

12. Kleber, W.; Makin, B. Triboelectric powder coating: A practical approach for industrial use. Part. Sci. Technol. 1998, 16, 43-53. [CrossRef]

13. Kleber, W.; Lang, A. Triboelectrically charged powder coatings generated by running through holes and slits. J. Electrost. 1997, 40, 237-240. [CrossRef]

14. Metcalf, K.A.; Wright, R.J. Xerography and electrostatic printing. Cartography 1957, 2, 67-81. [CrossRef]

15. Khan, M.K.I.; Schutyser, M.A.; Schroën, K.; Boom, R.M. Electrostatic powder coating of foods-State of the art and opportunities. J. Food Eng. 2012, 111, 1-5. [CrossRef]

16. Miao, P.; Balachandran, W.; Xiao, P. Characterization of $\mathrm{ZrO} 2$ and $\mathrm{SiC}$ ceramic thin films prepared by electrostatic atomization. J. Mater. Sci. 2001, 36, 2925-2930. [CrossRef]

17. Cooper, S.C.; Law, S.E. Electrostatic sprays for sunless tanning of the human body. IEEE Trans. Ind. Appl. 2006, 42, 385-391. [CrossRef]

18. Gaunt, L.F.; Hughes, J.F.; Harrison, N.M. Electrostatic deposition of charged insecticide sprays on electrically isolated insects. J. Electrost. 2003, 57, 35-47. [CrossRef]

19. Kacprzyk, R.; Pelesz, A. Wpływ elektryzacji cząstek aerozolu na proces pokrycia obiektu. Prz. Elektrotech. 2012, 88, 203-206.

20. Lake, J.R. The deposition of electrostatically charged sprays on parts of targets shaded from the spray. J. Agric. Eng. Res. 1988, 39, 9-18. [CrossRef]

21. Dante, E.T.; Gupta, C.P. Deposition studies of an electrostatic spinning disc sprayer. Trans. ASAE. 1991, 34, 1927-1934. [CrossRef]

22. Hernandez-Sierra, A.; Alguacil, F.J.; Alonso, M. Unipolar charging of nanometer aerosol particles in a corona ionizer. J. Aerosol Sci. 2003, 34, 733-745. [CrossRef]

23. Alguacil, F.J.; Alonso, M. Multiple charging of ultrafine particles in a corona charger. J. Aerosol Sci. 2006, 37, 875-884. [CrossRef]

24. Law, S.E. Embedded-electrode electrostatic-induction spray-charging nozzle: Theoretical and engineering design. Trans. ASAE. 1978, 21, 1096-1104.

25. Zhao, S.; Castle, G.P.; Adamiak, K. Comparison of conduction and induction charging in liquid spraying. J. Electrost. 2005, 63, 871-876. [CrossRef]

26. Kacprzyk, R.; Lewandowski, M. Post-dispersion electrification of droplets. J. Electrost. 2016, 79, 33-37. [CrossRef]

27. Kacprzyk, R.; Lewandowski, M. Post-dispersion electrification of droplets in a system with pneumatic atomization. J. Phys. Conf. Ser. 2011, 301, 012030. [CrossRef] 
28. Zhao, S.; Castle, G.P.; Adamiak, K. The effect of space charge on the performance of an electrostatic induction charging spray nozzle. J. Electrost. 2005, 63, 261-272. [CrossRef]

29. Maski, D.; Divaker, D. Effects of electrode voltage, liquid flow rate, and liquid properties on spray chargeability of an air-assisted electrostatic-induction spray-charging system. J. Electrost. 2010, 68, 152-158. [CrossRef]

30. Kacprzyk, R.; Żyłka, P. Efekt ekranowania podczas elektryzacji cząstek aerozoli w głowicach z naddźwiękowym przepływem gazu. Prz. Elektrotech. 2010, 86, 143-146.

31. Im, K.S.; Lai, M.C.; Yu, S.T.J.; Matheson, R.R. Simulation of spray transfer processes in electrostatic rotary bell sprayer. J. Fluids Eng. 2004, 126, 449-456. [CrossRef]

32. Patel, M.K.; Sharma, T.; Nayak, M.K.; Ghanshyam, C. Computational modeling and experimental evaluation of the effects of electrode geometry and deposition target on electrostatic spraying processes. Int. J. Comput. Appl. 2015, 124, 10-15.

33. Colbert, S.A.; Cairncross, R.A. A computer simulation for predicting electrostatic spray coating patterns. Powder Technol. 2005, 151, 77-86. [CrossRef]

34. Toljic, N.; Adamiak, K.; Castle, G.P.; Kuo, H.H.H.; Fan, H.T.C. 3D numerical model of the electrostatic coating process with moving objects using a moving mesh. J. Electrost. 2012, 70, 499-504. [CrossRef]

(C) 2020 by the authors. Licensee MDPI, Basel, Switzerland. This article is an open access article distributed under the terms and conditions of the Creative Commons Attribution (CC BY) license (http://creativecommons.org/licenses/by/4.0/). 